\title{
Optimal back-off point determination and controller weight selection for multivariate systems under finite-horizon control
}

\author{
Tim Spinner ${ }^{1}$, Babji Srinivasan ${ }^{2}$, Raghunathan Rengaswamy $y^{* 1,3}$ \\ ${ }^{1}$ Department of Chemical Engineering, Texas Tech University, Lubbock, TX, USA \\ ${ }^{2}$ Department of Chemical Engineering, IIT Gandhinagar, VGEC Campus, Chandkheda, Ahmedabad, 382424, India \\ ${ }^{3}$ Department of Chemical Engineering, Indian Institute of Technology - Madras, Chennai, 600036, India
}

\begin{abstract}
Plant economic performance is most often related to the operating point, specifically the mean values of the process variables; meanwhile, most existing performance assessment techniques involve examining the variances or covariances of the controlled variables. A combined approach is to determine the appropriate trade-off between variances of different process variables in order to operate the plant at the point that provides maximum economic benefit while satisfying the operating constraints. This problem is referred to as the minimum backed-off operating point selection, and previous works have formulated it as a non-convex constrained optimization problem. In the current work, a new technique is introduced that can provide the optimal plant operating point. Additionally, this method provides the weights for a finite horizon controller that results in the optimal trade-off in process variable variances that will allow satisfaction of the operating constraints at the optimal operating point. In this method, the plant and disturbance models for the given process are used to generate data representing possible trade-offs between process variable standard deviations. Employing a piecewise linear regression to describe the sample points of this standard deviations data allows for the operating point selection problem to be solved as a small number of linear programs. The advantages of this approach are demonstrated through the use of mathematical and simulation case studies.
\end{abstract}

Keywords: Multivariate control loop performance assessment; Optimal plant operating point; Generalized principal component analysis; Piece-wise linear regression

\section{Introduction}

In process systems, the manipulated inputs are typically bounded by physical constraints, such as the opening range of a control valve, while process outputs are more likely to be bounded by safety or quality constraints. These two types of operating restrictions are termed hard constraints and soft constraints, respectively. Temporary, infrequent violations of soft constraints occurring as part of the dynamic system response are often tolerated at some level, say for example, a controlled temperature that is allowed to exceed its upper bound for short periods.

It almost always occurs that higher profit operating regions occur at the operating boundary created by the intersection of constraints [1]. For single-input single-output (SISO) systems, this has led to the observation that decreasing the variance of the output allows the operating point to be moved closer to boundary, thus increasing the profitability [2]. This phenomenon is illustrated in Fig. 1 for the case of a variable $y$ whose mean value we wish to maximize, but whose value is limited by a hard constraint $y_{u b}$. Initially, $y$ has a distribution characterized by mean value $\bar{y}_{a}$ and standard deviation $\sigma_{a}$. If through some means this standard deviation could be reduced to $\sigma_{b}$, then $y$ could be moved closer to $y_{u b}$, allowing this variable to take on more profitable operating value $\bar{y}_{b}$.

\footnotetext{
${ }^{*}$ Corresponding author

Email address: raghur@iitm.ac. in (Raghunathan Rengaswamy*,1,3)
} 
This connection between output variance and profitability led to the creation of the minimum variance index (mvi) as a measure of the control system performance in SISO control loops [3]. The minimum variance index benchmarks the current variance of the controlled output against the theoretical lower bound of achievable output variance. The $m v i$ has seen wide adoption in the process industries, as its use provides a measure of performance that requires the use of only routine operating data along with knowledge of the process time delay in its calculation[4].

Due to the successful application of the minimum variance index to SISO systems, researchers have attempted to extend the idea of variance benchmarking of control loops to multivariate systems. Methods which rely on knowledge of the system interactor matrix [5, 6], as well as methods that do not require this device [7], were introduced in order to provide the theoretical minimum sum of output variances achievable for a given set of process and disturbance models. However, the utility of minimum variance benchmarking for multivariate systems is less clear than that for SISO systems, as decreasing the variance of one process variable can often cause other variables to increase in variance which may force the whole system to be further backed-off away from the optimal operating region. Even for SISO systems, the trade-off of variance between the controller output and process output should be considered in many cases [8].

Another way to look at the input and output variances, providing a clearer connection to plant economics, is as limitations on the choice of the plant operating point. The standard deviations of plant variables determine how close that the actual plant operating point can come to the optimal operating point that lies upon the boundary formed by operating constraints. Finding the optimal backed-off point is called the minimum backed-off operating point selection $[9,10]$. This usually involves transforming the original operating constraints on input and output values into a probabilistic form, wherein the constraint on each variable is written in terms of the standard deviation of that variable. Then the choice of operating point, along with the target variance of each process input and output, is selected within an optimization problem, with an objective function representing the process economics. A brief overview of the various approaches ( along with their limitations) used to solve the minimum backed-off operating point selection is discussed.

\subsection{Motivation $\mathcal{G}$ Important Contributions}

The work of Chmielewski and Manthanwar [11] along with that of Peng et al. [12] formulated the minimum backed-off operating point selection as a problem with a linear objective function with convex and reverse convex constraints. They required the use of a branch and bound solution technique to solve for the optimal operating point. Nabil et al. formulated the problem in a similar manner, and used an iterative solution technique to deal with the nonconvexity [13]. Zhao et al. considered a more restricted problem in which not all of the input and output variances were free to vary [14].Their formulation was valid for any model type provided that the control weightings describing the trade-off between the variances of the process variables were predetermined.

The current work seeks to consider the determination of controller weights as part of the overall optimization problem. However, unlike the aforementioned earlier works, herein the problem's constraints are linearized in order to eliminate the need for non-convex or iterative programming. The method proposed is capable of minimizing the variance of input and output variables in an integrated manner, hence suitable for multivariable process. Further, the proposed method obtains the solution to the optimization problem by solving a number of linear optimization tasks, one of the important contributions of this work. This is possible because of the use of the ideas of gpca (generalized principal component analysis) and k-DOP (discrete oriented polytopes). These ideas have not been used before in multivariate controller performance assessment.

The proposed methodology requires a multivariate finite impulse response model of the plant and the disturbance affecting the process. A large sample of the closed-loop standard deviations of the process variables is then generated for the system under analysis. A piecewise linearization and subspace bounding procedure for this generated data is then used to generate linear equality and inequality constraints to describe the relationship between the standard deviations of different process variables. The economically optimal operating point can then be determined by solving a small set of linear programs. Section 2 provides an introduction to some of the existing data handling and modelling techniques (Generalized Principal Component Analysis (gpca) and Discrete Oriented Polytopes (DOP)) applied in later sections. Section 3 contains the formulation of the operating point and variance tradeoff problem to be solved. Section 4 contains the proposed method for solving this problem. Section 5 contains both mathematical and simulation examples to demonstrate the proposed approach. Finally, conclusions are provided in Section 6. 


\section{Preliminaries}

This section introduces several techniques that will be applied in later sections. The first two techniques are for piecewise linearization and bounding of a multidimensional surface, while the last is useful for modeling step-type process disturbances.

\subsection{Generalized principle component analysis}

Generalized principle component analysis ( $g p c a$ ) is a technique that allows for simultaneous data segmentation and model estimation for a data set that has come from a collection of linear subspaces of potentially unknown number and dimension [15]. gpca formulates either a set of factorable polynomials or else a basis for this set, allowing for an algebraic solution for the determination of the unknown data subspaces. A statement of the problem which gpca serves to solve is as follows: Given a data set $\left\{x_{j}\right\}_{j=1}^{N_{p}}$ consisting of $N_{p}$ points existing in $\mathbb{R}^{K}$, drawn from $k$ linear subspaces $\left\{S_{i} \subseteq \mathbb{R}\right\}_{i=1}^{k}$ of dimension $K_{i}=\operatorname{dim}\left(S_{i}\right)$, identify the following [15]:

1. the number of subspaces $(k)$ and the dimension of each $\left(K_{i}\right)$

2. a basis for each subspace $S_{i}$

3. the membership of each data point $x_{j}\left(j=1 \ldots N_{p}\right)$ in one of the subspaces $S_{i}$.

Fig. 2 shows the outcome of this procedure for an illustrative example. In Fig. 2(a) data is plotted on its original set of axes. In Fig. 2(b) three gpca-determined subspaces are plotted that are proposed to represent the data. Subspaces $S_{1}$ and $S_{2}$ are each of dimension 1, while $S_{3}$ is of dimension 2. In addition to giving the number of subspaces and their basis vectors, gpca will also provide the membership of each point. In Fig. 2(c), the data points are projected onto their respective subspaces, and the transformed points are shown in the local subspace coordinates.

The gpca algorithm works by formulating the segmentation and projection problem as an algebraic problem described by a polynomial and its derivatives. gpca allows for the subspaces to have arbitrary intersections with each other and can be used alone or as an initialization method for other segmentation techniques such as k-subspaces or expectation maximization [16]. For details on the exact steps of the gpca procedure, the reader is referred to the works by Vidal et al. $[16,15]$. In Section 4, it is proposed to use the polynomial differentiation algorithm implementation of gpca in order to determine a piecewise linear approximation to a surface in high dimensional space.

\subsection{Discrete oriented polytopes}

Discrete oriented polytopes have been widely applied in the field of computer graphics for use in performing fast collision detection between moving objects [17]. A k-DOP is a discrete oriented polytope that is constructed from $k$ hyperplanes. Besides bounding of computer graphics objects, a k-DOP can be constructed to bound any collection of multidimensional data. For bounding a set of data points with a k-DOP, in each of $\kappa$ directions, the maximum projected magnitude of data projected onto that direction gives the point of intersection for the hyperplane normal to that direction. Fig. 3 illustrates the construction of a k-DOP on an example data set. The number and orientation of the bounding hyperplanes is user-defined, where the only requirement is that the choice of hyperplanes is sufficient to form a closed bounding volume.

In Section 4, the use of gpca provides a piecewise linear approximation to a bounded set of data in high dimension; however, the hyperplanes that are generated by gpca for this purpose are unbounded and extend to infinity. Therefore it is proposed to project the original data set onto the piecewise linear subspaces determined via $g p c a$, and then use k-DOPs upon each set of projected data, thus providing a bounded piecewise linear approximation to the surface described by the original data set.

\subsection{Modeling of step-type disturbances}

Processes are often affected by step type disturbances that last much longer than the controller time step. Disturbances of this type have been termed 'deterministic', but, as the value of the disturbance at any future time cannot be predicted with certainty, they are also of stochastic nature. One common way to represent step disturbances as stochastic processes is the use of an integrated white noise model; however, a disturbance following this model will become unbounded as $t \rightarrow \infty$ [18]. This means that the disturbance cannot be canceled by a bounded set of input moves, and either the input or output of the process will become unbounded when this disturbance model is used. 
In order to avoid this unbounded behavior, a stochastic discrete-time model for step disturbances similar to the one defined for pulses in chapter 3 of [19] is used in this work. Let $T_{c}$ denote the controller sampling time, and suppose that the disturbance has a mean time between step changes of $\mu_{s}$ samples, where $\mu_{s}$ is integer-valued. Then the disturbance model is

$$
d_{t}= \begin{cases}a_{t} & \text { if } t \in\left\{\mu_{s}, 2 \mu_{s}, 3 \mu_{s}, \ldots\right\} \\ d_{t-1} & \text { otherwise }\end{cases}
$$

where $a_{t}$ is a Gaussian white noise process with mean zero. Here we consider the process $a_{s, t}$ with $a_{s, t}=a_{t}$ for every $\mu_{s}$ and zero at all other samples. Then the step-type disturbance can be written in finite response filter form,

$$
\begin{aligned}
d_{t} & =f\left(z^{-1}\right) a_{s, t} \\
& =\left(1+z^{-1}+\cdots+z^{-\left(\mu_{s}-1\right)}\right) a_{s, t}
\end{aligned}
$$

where $z^{-1}$ is the backshift operator. With the above representation, if the original process $a_{t}$ has variance $\sigma_{a}^{2}$, then the sampled version $a_{s, t}$ will have variance $\sigma_{a}^{2} / \mu_{s}[19]$.

For a multivariate system, if vector $d_{t}$ contains $n_{d}$ step disturbances, denoted $d_{t, i}, i=1 \ldots n_{d}$, each having a respective mean step length of $\mu_{s, i}$ samples, then the $(i, i)^{\text {th }}$ entry of multiple-input multiple-output (MIMO) diagonal step filter $F\left(z^{-1}\right)$, can be expressed

$$
F_{i i}\left(z^{-1}\right)=1+z^{-1}+\cdots+z^{-\left(\mu_{s, i}-1\right)} .
$$

The input is vector white noise process $a_{s, t}$ of length $n_{d}$, wherein each element is constructed by sampling white noise process $a_{t, i}$ according to respective step length $\mu_{s, i}\left(i=1 \ldots n_{d}\right)$. Each of the diagonal entries of $\Sigma_{a}$, the covariance matrix of $a_{s, t}$, can be computed according to

$$
\left(\Sigma_{a}\right)_{i i}=\sigma_{a, i}^{2} / \mu_{s, i}
$$

where $\sigma_{a, i}^{2}$ is the variance of the $i_{t h}$ scalar white noise process sampled in order to construct vector process $a_{s, t}$. Finally, the resulting vector of step disturbance processes can be simply written

$$
d_{t}=F\left(z^{-1}\right) a_{s, t}
$$

Matrix $\Sigma_{a}$ and the disturbance model of Eqns. $3 \& 5$ will be used in the following sections to compute the closed-loop variances of process variables as these quantities depend on the properties of the disturbance.

\section{Problem Formulation}

In this section, an optimization problem is formulated for choosing the operating point for a certain class of constrained linear systems subjected to stochastic disturbances. The operating point can be expressed as a pair of vectors, $(\bar{y}, \bar{u})$, where $\bar{y}$ contains the mean value of each of the process outputs while $\bar{u}$ contains the mean values of the controller outputs. The present method requires that the system under analysis is able to be described with linear finite impulse models of both the process and disturbance, with each disturbance driven by a Gaussian white noise process, as shown in Fig. 4. In that case, the process output can be expressed as

$$
y_{t}-y_{o}=H\left(z^{-1}\right)\left(u_{t}-u_{o}\right)+N\left(z^{-1}\right) d_{t}
$$

where $y_{t}$ and $u_{t}$ are the process output and controller output vectors at sample time $t,\left(y_{o}, u_{o}\right)$ is the linearization point around which Eqn. 6 is valid, $d_{t}$ is a disturbance of the type in Eqn. 5, and $H\left(z^{-1}\right)$ and $N\left(z^{-1}\right)$ are multivariate discrete finite impulse response models of the plant and disturbance, respectively. The plant and disturbance models can be expressed as $H\left(z^{-1}\right)=\sum_{i=0}^{N_{H}} H_{i} z^{-i}$ and $N\left(z^{-1}\right)=\sum_{i=0}^{N_{D}} N_{i} z^{-i}$, respectively, with $N_{H}$ and $N_{D}$ being the order of each finite impulse response model, while $H_{i}$ and $N_{i}$ are the matrix coefficients of the respective models. It is a standard assumption that there is a delay separating the change of any controller output from its effect on the plant output, so that $H_{0}=\mathbf{0}$. 
Eqn. 6 can also be expressed in terms of deviation variables as

$$
y_{t}^{\Delta}=H\left(z^{-1}\right) u_{t}^{\Delta}+N\left(z^{-1}\right) d_{t}
$$

where $y_{t}^{\Delta}=y_{t}-y_{o}$ and $u_{t}^{\Delta}=u_{t}-u_{o}$.

Within the process industries, it often occurs that input variables are restricted by physical constraints, such as the amount that a valve can open and close, whereas output variables are constrained by quality, safety, or operational concerns that allow for temporary violations; these restrictions are termed "hard" constraints and "soft" constraints, respectively. As in previous works on operating point selection [12, 14], the current method uses a probabilistic description of constraint satisfaction. In this way, soft constraints on each of the outputs $y_{i}\left(i=1 \ldots n_{y}\right)$ can be written as

$$
\operatorname{Pr}\left\{y_{l b, i} \leq y_{t, i} \leq y_{u b, i}\right\} \geq 1-\alpha_{i}
$$

where $\operatorname{Pr}\{\cdot\}$ is the probability operator, $y_{l b, i}$ and $y_{u b, i}$ are the lower and upper bounds on output $i$, and $\alpha_{i}$ is the permissible level of constraint violation. If $y_{t, i}$ is generated from a Gaussian process having mean $\bar{y}_{i}$ and standard deviation $\sigma_{y, i}$, the previous constraint will be satisfied if

$$
y_{l b, i}+z_{y, i} \sigma_{y, i} \leq \bar{y}_{i} \leq y_{u b, i}-z_{y, i} \sigma_{y, i}
$$

where $z_{y, i}$ is the z-score associated with violation level $\alpha_{i} / 2$. For example, if $y_{t, i}$ is required to stay within its bounds $\left(y_{l b, i}, y_{u b, i}\right) 95 \%$ of the time, then $\alpha_{i}$ is 0.05 and the corresponding $z_{y, i}$ equals 1.96 . In this case, $y_{t, i} \leq y_{l b, i}$ is expected to occur less than $2.5 \%$ of the time, with this same violation rate expected for $y_{t, i} \geq y_{u b, i}$, leading to the satisfaction of Eqn. 8.

For consideration of hard constraints during operating point selection and controller design, a similar framework as above can be used. While this probabilistic formulation cannot enforce hard constraints, it can make violations of them unlikely enough that performance differences versus the real process are negligible. If each set of lower and upper bounds, $\left(u_{l b, j}, u_{u b, j}\right)$, for controller output $u_{t, j}\left(j=1 \ldots n_{u}\right)$ is written as

$$
u_{l b, j}+z_{u, j} \sigma_{u, j} \leq \bar{u}_{j} \leq u_{u b, j}-z_{u, j} \sigma_{u, j}
$$

then setting $z_{u, j}$ equal to 3 , for example, ensures that the violations of the bounds are predicted to occur less than $0.3 \%$ of the time. In practice, actuator saturation will occur instead of constraint violations, but the low frequency of occurrence should allow for negligible differences between the real and modeled processes.

For a system which allows for this description of process, disturbance, and constraints, the problem of optimizing an economic objective function with linear profit dependency on the mean values of the process variables can be expressed as

$$
\begin{aligned}
& \max _{\bar{y}, \bar{u}, \sigma_{u}, \sigma_{y}} J=c_{y}^{T} \bar{y}-c_{u}^{T} \bar{u} \\
& \text { subject to: } y_{l b}+z_{y} \sigma_{y} \leq \bar{y} \leq y_{u b}-z_{y} \sigma_{y} \\
& u_{l b}+z_{u} \sigma_{u} \leq \bar{u} \leq u_{u b}-z_{u} \sigma_{u} \\
& y_{t}^{\Delta}=H\left(z^{-1}\right) u_{t}^{\Delta}+N\left(z^{-1}\right) d_{t} \\
& \left(\bar{y}-y_{o}\right)=K\left(\bar{u}-u_{o}\right)
\end{aligned}
$$

where $J$ is a measure of economic performance, $c_{y}$ is a vector of coefficients giving value to the level of each output variable, $c_{u}$ is a vector of cost coefficients associated with each controller output level, $K$ is the steady state gain, while $y_{l b}, z_{y}, y_{u b}, \sigma_{y}, u_{l b}, z_{y}, u_{u b}, \sigma_{u}$ are vectors of the associated indexed quantities defined in Eqns. 8-10. This linear objective function is usually applicable to cases where the output represents the product with $c_{y}$ denoting its cost while the input $u$ is the total energy ( $c_{u}$ is the associated cost) incurred to develop the product. This is one of the widely used objective functions in linear programming problems. However, this linear objective function could be modified depending on the control problem at hand, provided the model and other constraints in the above optimization problem of Eqn. 11 are valid. For instance, the objective function could be modified to minimizing the sum squared deviation of the process and controller outputs (as discussed in Nabil et al. [13]) from a reference value of interest.

The optimization problem of Eqn. 11 is not yet in a solvable format since the connection between the process model and the process variable standard deviations is not explicit. The following section details how to generate a surface of Pareto optimal standard deviation values, $\sigma_{y}$ and $\sigma_{u}$, from the impulse response model of Eqn. 7 and to furthermore create a bounded piecewise linear approximation of this curve so that the optimization problem of Eqn. 11 can be solved as a series of linear programs. 


\section{Solution Method}

This section first details how a relationship among and between the entries in the vectors $\sigma_{y}$ and $\sigma_{u}$ can be generated. Following this, a procedure for the piecewise linearization of this relationship is introduced. This novel method allows the optimization of Eqn. 11 to be obtained via the solution of a small set of linear programs.

\subsection{Generating Pareto Optimal Standard Deviations}

The objective and each of the constraints in Eqn. 11 are linear, except for the process description of Eqn. 7. Also, it is not yet explicit how the allowable set of output and input variances $\left(\sigma_{y}, \sigma_{u}\right)$ are to be generated. This section will show how the set of admissible $\sigma_{y}$ and $\sigma_{u}$ are obtained as part of the solution to a set of finite horizon control problems relevant to the process of Eqn. 7. Much of the following developments in this subsection are based on the framework used for minimum variance performance benchmarking introduced by Ko \& Edgar [20], but are presented with appropriate modification for the different problem now considered.

In this section, it is desired to solve a control problem for the system of Eqn. 7. Consider a finite horizon length control law which selects $N_{U}$ moves for each controller output, which means that the plant response can be calculated for $N_{H}+N_{U}$ total time steps. Now considering the disturbance model of Eqn. 5 along with the disturbance term in Eqn. 7, the step filter $F\left(z^{-1}\right)$ has $N_{F}=\max _{j \in 1 \ldots n_{d}}\left(\mu_{s, j}\right)$ coefficients which, when convoluted with filter $N\left(z^{-1}\right)$, gives a total disturbance response of length $N_{D}+N_{F}$ time steps. To create equivalent length plant and disturbance responses, take $N=\max \left(N_{U}+N_{H}, N_{F}+N_{D}\right)$, then append zero coefficients to either the plant response filter $H\left(z^{-1}\right)$ or disturbance response filter $N\left(z^{-1}\right)$ so that finally plant and disturbance both have equal response length $N=N_{U}+N_{H}=N_{F}+N_{D}$. Also, it will be useful in the following analysis to redefine the disturbance impulse response filter in Eqn. 7 to be driven by noise $a_{s, t}$ instead of step disturbance $d_{t}$. To do so, the disturbance filter $N^{\prime}\left(z^{-1}\right)$ is defined by

$$
\begin{aligned}
N^{\prime}\left(z^{-1}\right) & =N\left(z^{-1}\right) * F\left(z^{-1}\right) \\
& =\sum_{i=0}^{N_{D}} N_{i} z^{-i} \sum_{j=0}^{\max \left(N-i, N_{F}\right)} F_{j} z^{-j}=\sum_{\ell=0}^{N-1} N_{\ell}^{\prime} z^{-\ell}
\end{aligned}
$$

where the coefficients of filter $N^{\prime}\left(z^{-1}\right)$ must be calculated according to the convolution product.

Since it is desired to compute the variances of both plant inputs and outputs, it is useful to augment the plant and disturbance models with the plant inputs treated as additional outputs. The augmented model takes the form

$$
\begin{aligned}
\widetilde{y}_{t}=\left[\begin{array}{l}
y_{t}^{\Delta} \\
u_{t}^{\Delta}
\end{array}\right] & =\left[\begin{array}{cc}
H\left(z^{-1}\right) & \mathbf{0} \\
\mathbf{0} & \boldsymbol{I}
\end{array}\right] u_{t}^{\Delta}+\left[\begin{array}{c}
N^{\prime}\left(z^{-1}\right) \\
\mathbf{0}
\end{array}\right] a_{s, t} \\
& =\widetilde{H}\left(z^{-1}\right) u_{t}^{\Delta}+\widetilde{N}\left(z^{-1}\right) a_{s, t}
\end{aligned}
$$

where $\widetilde{H}\left(z^{-1}\right)$ is the augmented plant filter, and $\widetilde{N}\left(z^{-1}\right)$ is the augmented disturbance filter, which can be expressed as $\widetilde{H}\left(z^{-1}\right)=\sum_{i=0}^{N_{H}} \widetilde{H}_{i} z^{-i}$ and $\widetilde{N}\left(z^{-1}\right)=\sum_{i=0}^{N-1} \widetilde{N}_{i} z^{-i}$, respectively. Note again that $u_{t}^{\Delta}$ and $y_{t}^{\Delta}$ represent deviation variables and not increments in $y_{t}$ and $u_{t}$.

From the MIMO plant filter impulse coefficients $\widetilde{H}_{i}$ that make up plant model $\widetilde{H}\left(z^{-1}\right)$, the dynamic matrix $G_{N}$, can be defined as:

$$
G_{N}=\left[\begin{array}{cccc}
\widetilde{H}_{0} & 0 & 0 & 0 \\
\vdots & \ddots & 0 & 0 \\
\widetilde{H}_{N_{H}} & \cdots & \widetilde{H}_{0} & 0 \\
0 & \widetilde{H}_{N_{H}} & \cdots & \widetilde{H}_{0} \\
0 & 0 & \widetilde{H}_{N_{H}} & \cdots \\
\vdots & \ddots & \cdots & \vdots \\
0 & \cdots & 0 & \widetilde{H}_{N_{H}}
\end{array}\right]
$$


Also, matrix $D_{N}=\left[\begin{array}{c}\widetilde{N}_{0} \\ \vdots \\ \widetilde{N}_{N-1}\end{array}\right]$ can be constructed from disturbance filter impulse coefficients $\widetilde{N}_{i}$ describing the total disturbance effect resulting from a single white noise vector impulse $a_{0}$ from sequence $a_{s, t}$. Therefore, the effect of $a_{0}$ on augmented output $\widetilde{y}_{t}$ can be written

$$
\boldsymbol{y}_{N}^{\mathbf{\Delta}}=G_{N} \boldsymbol{u}_{N}^{\mathbf{\Delta}}+D_{N} a_{0}
$$

where $\boldsymbol{y}_{N}^{\Delta}=\left[\begin{array}{c}\widetilde{y}_{0} \\ \vdots \\ \widetilde{y}_{N-1}\end{array}\right]$ and $\boldsymbol{u}_{N}^{\Delta}=\left[\begin{array}{c}u_{0}^{\Delta} \\ \vdots \\ u_{N_{U}-1}^{\Delta}\end{array}\right]$

Suppose it is desired to achieve the minimum of a weighted sum of input and output variances

$$
\sigma_{W}^{2}=\sum_{i=1}^{n_{y}} w_{y, i} \sigma_{y, i}^{2}+\sum_{j=1}^{n_{u}} w_{u, j} \sigma_{u, j}^{2}
$$

through proper manipulations of $u_{t}^{\Delta}$. First, letting $w_{y}$ and $w_{u}$ be the vectors whose entries are $w_{y, i}$ and $w_{u, j}$, respectively, construct weighting matrix $W=\operatorname{diag}\left(\left[\begin{array}{ll}w_{y}^{T} & w_{u}^{T}\end{array}\right]\right)$ along with matrix $W_{N}$, which contains $N$ repetitions of $\mathrm{W}$ on its block diagonal. Then, minimizing the weighted sum of variances is equivalent to minimizing the expectation $\mathbb{E}\left(\boldsymbol{y}_{N}^{\Delta^{T}} W_{N} \boldsymbol{y}_{N}^{\Delta}\right)$. The solution of this weighted linear least squares problem is well known, with the optimal $\boldsymbol{u}_{N}^{\Delta}$ given by

$$
\begin{aligned}
\boldsymbol{u}_{N}^{\Delta} & =\boldsymbol{U}_{N} a_{0}=\left[\begin{array}{c}
U_{0} \\
\vdots \\
U_{N_{U}-1}
\end{array}\right] a_{0} \\
& =-\left(G_{N}^{T} W_{N} G_{N}\right)^{+} G_{N}^{T} W_{N} D_{N} a_{0}
\end{aligned}
$$

where $\boldsymbol{U}_{N}$ is the projection matrix $-\left(G_{N}^{T} W_{N} G_{N}\right)^{+} G_{N}^{T} W_{N} D_{N}$, and each coefficient $U_{i}, i=0 \ldots N_{U}-1$, is of size $n_{u} \times 1$. If the values of the random shocks $a_{s, t}$ are either known or can be estimated, the coefficients $U_{i}$ can be used to construct a feedforward controller. Due to the statistical independence of the white noise sequence $a_{s, t}$ in time, the principle of superposition holds [20], and this controller takes the form $u_{t}=-\sum_{i=0}^{N_{U}-1} U_{i} a_{s, t-i}$. Similarly, the closed-loop response to shock $a_{0}$ can be written as

$$
\begin{aligned}
\boldsymbol{y}_{N}^{\Delta} & =\boldsymbol{Y}_{N} a_{0}=\left[\begin{array}{c}
Y_{0} \\
\vdots \\
Y_{N-1}
\end{array}\right] a_{0} \\
& =\left[I-G_{N}\left(G_{N}^{T} W_{N} G_{N}\right)^{+} G_{N}^{T} W_{N}\right] D_{N} a_{0}
\end{aligned}
$$

where each $Y_{i}$ is a $\left(n_{y}+n_{u}\right) \times 1$ coefficient. Then, the response to sequence $a_{s, t}$ can be computed via the filter equation $y_{t}=-\sum_{i=0}^{N-1} Y_{i} a_{s, t-i}$.

It follows that the covariance matrix $\Sigma_{\widetilde{y}}$ of the augmented process output $\widetilde{y}$ that corresponds to the minimized $\sigma_{W}^{2}$ is computed through

$$
\Sigma_{\widetilde{y}}=\sum_{i=0}^{N-1} Y_{i} \Sigma_{a} Y_{i}^{T}
$$

with this result due to the statistical independence of any terms within white-noise sequence $a_{s, t}$, which provides $\operatorname{cov}\left(a_{s, t-i}, a_{s, t-j}\right)=\mathbf{0}$ [8]. Finally, the vectors $\sigma_{y}$ and $\sigma_{u}$ can be obtained by taking the square root of the diagonal elements of $\Sigma_{\tilde{y}}$. The standard deviation points generated in this manner are Pareto optimal, meaning that lowering the value of any entry in $\sigma_{y}$ or $\sigma_{u}$ requires that one or more of the other values within these vectors must be increased. 
To create the Pareto optimal surface of $\left(\sigma_{y}, \sigma_{u}\right)$ values, this procedure should be carried out repeatedly over a wide range of values of the weights within matrix $W$ (by varying the entries in $w_{y}$ and $w_{u}$ from $10^{-4}$ to $10^{4}$, for example). The resulting surface of these points in $\left(\sigma_{y}, \sigma_{u}\right)$ space represents a description of the possible trade-offs in variation between different process variables.

\subsection{Linearization of $\left(\sigma_{y}, \sigma_{u}\right)$ Space}

The Pareto optimal standard deviations will create an open surface within a space over $\left(n_{u}+n_{y}\right)$ dimensions, where $n_{u}$ is the number of inputs and $n_{y}$ the number of outputs. In order to proceed, it is proposed to create a piecewise-linear approximation of the Pareto surface. For this purpose, the technique of Generalized Principle Component Analysis ( $g p c a$ ) is employed [15]. For data points in the $K$-dimensional space of real numbers, gpca can determine the optimal division of these points into a set of linear subspaces $\left\{S_{i}\right\}_{i=1}^{k}$, along with simultaneous determination of the basis for each linear subspace.

First define $\sigma=\left[\begin{array}{l}\sigma_{y} \\ \sigma_{u}\end{array}\right]$ and let $\left\{\sigma_{j}\right\}_{j=1}^{N_{p}}$ be a set of $\sigma$ vectors generated using the procedure of the previous section, where $N_{p}$ is the total number of standard deviation samples generated. This set is assumed to be representative of the surface of Pareto optimal $\sigma$ values. Then, the gpca technique can be used to determine the number of linear subspaces $k$ and the basis vectors for each of these subspaces that best represents the $\sigma$ data. Using the subspace basis vectors and membership of each data point, both of which are determined via the gpca procedure, each point in the set $\left\{\sigma_{j}\right\}_{j=1}^{N_{p}}$ is projected onto its assigned subspace. If $V_{i}$ is the matrix whose columns are the basis vectors of the subspace $S_{i}$ of the standard deviation data, then

$$
P_{V, i}=V_{i}\left(V_{i}^{T} V_{i}\right)^{+} V_{i}^{T}
$$

is the projection matrix for $V_{i}$, where $\left(V_{i}^{T} V_{i}\right)^{+}$denotes the pseudoinverse of the product $V_{i}^{T} V_{i}$. Therefore, if point $\sigma_{j}$ was determined to belong to subspace $i$, its projection onto the linear subspace $S_{i}$, expressed in the original coordinates $\left(\sigma_{y}, \sigma_{u}\right)$, is given by

$$
\widetilde{\sigma}_{j}=P_{V, i}\left(\sigma_{j}-\mu_{\sigma, i}\right)+\mu_{\sigma, i}
$$

where $\mu_{\sigma, i}$ is the mean of the $\sigma_{j}$ belonging to subspace $i$. Therefore, it follows that any $\sigma$ belonging to the $i^{\text {th }}$ linearized subspace must satisfy:

$$
\left(P_{V, i}-I\right)\left(\sigma-\mu_{\sigma, i}\right)=0
$$

With Eqn 22, a set of constraints has been formulated that restricts $\sigma$ of Eqn 11 to a set of linear subspaces $\left\{S_{i}\right\}_{i=1}^{k}$. However, these subspaces will be unbounded and thus allow for $\sigma$ points far outside of the original Pareto surface. To restrict $\sigma$ to a bounded set that represents the original standard deviation data, the k-DOPs method introduced in Sec. 2.2 is applied to each of the gpca-determined subspaces. If vector $s$ is a chosen bounding direction within subspace $S_{i}$, then the boundaries on $\sigma$ in directions $s$ and $-s$ can be expressed as:

$$
\min _{\sigma_{j} \in S_{i}}\left(s^{T} P_{V, i} \sigma_{j}\right) \leq s^{T} P_{V, i} \sigma \leq \max _{\sigma_{j} \in S_{i}}\left(s^{T} P_{V, i} \sigma_{j}\right)
$$

where the $\sigma_{j}$ are the Pareto optimal standard deviation points determined to belong to subspace $i$.

Then, after this procedure is carried out over a set of directions $\{s\}$ on each subspace $S_{i}$, the model satisfaction constraint, Eqn 7, is transformed into a pair of matrix equality and inequality constraints that describe a series of hyperplanes approximating the $\sigma$ relation in addition to the bounds of each hyperplane, as follows:

$$
A^{i}\left[\begin{array}{l}
\sigma_{y} \\
\sigma_{u}
\end{array}\right] \leq b^{i} \quad C^{i}\left[\begin{array}{l}
\sigma_{y} \\
\sigma_{u}
\end{array}\right]=d^{i}
$$

where $A^{i}, b^{i}, C^{i}, d^{i}$ are coefficient matrices corresponding to the $i^{\text {th }}$ linearized subspace, $i=1 \ldots k$, where $k$ is the number of subspaces identified, and

$$
\begin{array}{rlrl}
A^{i} & =\left[\begin{array}{c}
s^{T} P_{V, i} \\
-s^{T} P_{V, i}
\end{array}\right] \\
C^{i} & =P_{V, i}-I & b^{i} & =\left[\begin{array}{c}
\max _{\sigma_{j} \in S_{i}}\left(s^{T} P_{V, i} \sigma_{j}\right) \\
-\min _{\sigma_{j} \in S_{i}}\left(s^{T} P_{V, i} \sigma_{j}\right)
\end{array}\right] \\
& d^{i}=\left(P_{V, i}-I\right) \mu_{\sigma, i}
\end{array}
$$


with the result that the surface of $\sigma$ data has been approximated with a set of linear and bounded subspaces. At this point, the optimal operating point and $\sigma$ can be found by solving Eqn. 11, with the substitution of Eqn. 24 in the place of model Eqn. 7. This proposed framework for piecewise linearization and optimization is summarized in Fig. 5. Detailed examples, illustrating how this procedure is used, follow in the next section.

Remark 4.1. In the proposed approach, controller weights are obtained by mapping the $\sigma\left[\sigma_{y}, \sigma_{u}\right]$ space to the space of controller weights. With the help of this mapping, the optimal controller weights are obtained based on the optimal $\sigma$ values obtained by solving the equation 11 as per the method outlined in Figure 5. In the proposed approach, controller weights are obtained by mapping the variance space to the space of controller weights. With the help of this mapping, the optimal controller weights can always be obtained in the least squares sense. It should be noted that since approximations are used (whether piece-wise or nonlinear), there is no guarantee that controller weights are globally optimal for the particular values of the variance. Nonlinear mapping (like neural networks used in section 5 and other Kernel methods) approaches might perform better in some specific cases.

Remark 4.2. The proposed approach tackles the optimization problem defined in equation 11 using an approximation of the $\sigma$ space resulting in a solution using a number of linear optimization tasks. In this sense, the proposed approach is numerically efficient compared to several other approaches discussed earlier. However, theoretically, there is no guarantee that the proposed approach will yield globally optimal results.

\section{Examples}

In this section, two case studies are presented which demonstrate the use of the proposed operating point and controller selection technique. In the first, the calculations described in Sec. 4 are presented for a simple 2-input 2output discrete time plant model. In the second example, the method is applied to a 3-input 3-output continuous-time simulation model.

\subsection{Wood E Berry Column Model}

The first system studied is a discrete approximation of the Wood \& Berry column model [21] with additional transfer functions defined to allow for two output disturbances. The plant $H\left(z^{-1}\right)$ and disturbance $N\left(z^{-1}\right)$ transfer functions used were

$$
H\left(z^{-1}\right)=\left[\begin{array}{ll}
\frac{0.744 z^{-2}}{1-0.9419 z^{-1}} & \frac{-0.8789 z^{-4}}{1-0.9535 z^{-1}} \\
\frac{-1.302 z^{-4}}{1-0.9329 z^{-1}} & \frac{-0.5786 z^{-8}}{1-0.9123 z^{-1}}
\end{array}\right]
$$

and

$$
N\left(z^{-1}\right)=\left[\begin{array}{cc}
\frac{2 z^{-1}}{1-0.8 z^{-1}} & \frac{0.9}{\left(1-0.7 z^{-1}\right)\left(1-0.7 z^{-1}\right)} \\
\frac{0.9}{\left(1-0.7 z^{-1}\right)\left(1-0.7 z^{-1}\right)} & \frac{2 z^{-1}}{1-0.8 z^{-1}}
\end{array}\right]
$$

The disturbances $d_{1}$ and $d_{2}$, which are the inputs to noise filter $N\left(z^{-1}\right)$, were of the form of Eqn. 2 each with a mean step time $\mu_{s}=20$. The noise covariance was $\Sigma_{a}=\left[\begin{array}{cc}0.125 & 0 \\ 0 & 0.125\end{array}\right]$. The proposed method requires transformation of the plant and disturbance models to finite impulse response form, and so the multivariate discrete transfer function matrices $H\left(z^{-1}\right)$ and $N\left(z^{-1}\right)$ were approximated by $N_{H}=100$ and $N_{D}=130$ impulse response coefficients, respectively. A control horizon of $N_{U}=50$ was selected, leading to the closed-loop disturbance impulse response between each disturbance $d_{i}$ and output $y_{i}$ to have 150 coefficients.

The linearization point was $\left[y_{1}, y_{2}, u_{1}, u_{2}\right]^{T}=[90,90,55,55]^{T}$, while each input was constrained to operate within the (hard) bounds $\left(u_{l b}, u_{u b}\right)=(0,60)$ and each process output was required to stay within the bounds $\left(y_{l b}, y_{u b}\right)=$ $(0,100)$ at least $95 \%$ of the time.

The objective function to be optimized was:

$$
\max _{\bar{y}, \bar{u}, \sigma_{u}, \sigma_{y}} J=c_{y} \bar{y}-c_{u} \bar{u}=\left[\begin{array}{ll}
5 & 5
\end{array}\right]\left[\begin{array}{l}
y_{1}^{*} \\
y_{2}^{*}
\end{array}\right]-\left[\begin{array}{ll}
2 & 2
\end{array}\right]\left[\begin{array}{l}
u_{1}^{*} \\
u_{2}^{*}
\end{array}\right]
$$


The procedure of Sec. 4.1 was used to minimize the weighted sum $w_{y, 1} \sigma_{y, 1}^{2}+w_{y, 2} \sigma_{y, 2}^{2}+w_{u, 1} \sigma_{u, 1}^{2}+w_{u, 2} \sigma_{u, 2}^{2}$ repeatedly, wherein each of the weights $w_{y, 1}, w_{y, 2}, w_{u, 1}, w_{u, 2}$ were varied in the range $10^{-4}$ to $10^{4}$, and approximately 35000 points in $\left(\sigma_{u, 1}, \sigma_{u, 2}, \sigma_{y, 1}, \sigma_{y, 2}\right)$ space were obtained. Subspaces of the resulting 4-dimensional space are shown in Fig. 6(a-c).

By following the procedure in Fig. 5, the original $\left(\sigma_{u, 1}, \sigma_{u, 2}, \sigma_{y, 1}, \sigma_{y, 2}\right)$ space was then approximated in the form of $k=7$ piecewise linear and bounded spaces. Fig. 6(d) shows each $\sigma$ point of 6(c) projected onto its gpca-identified subspace. There is minor distortion of the $\sigma$ data observable, but the general bounds of the data are preserved. The gpca and k-DOPs procedures led to equations of the form Eqn. 24 for each linear subspace. The optimization of Eqn. 11 with objective function defined by Eqn. 26 was carried out once for every linear space, then the results for each were compared in order to select the optimal operating point and associated controller weights. In the following, this way of determining controller weights via the technique proposed in this work is referred to as Case I or the "optimized case". The resulting closed-loop disturbance impulse response of each of the process variables to white noise process $a_{s, t}$ are shown in Fig. 7 for this case. It is observed that the variables $y_{1}$ and $y_{2}$ have significant variation in their response for 10-20 time steps, whereas the form of matrix $H\left(z^{-1}\right)$ suggests a deadbeat control response could be achieved in as little as 4 time steps.

Therefore, the case traditionally referred to as "minimum variance" or "singular LQ" control was also considered for comparison against the present technique. In minimum variance control, the controller weights are set as $w_{y, 1}=$ $w_{y, 2}=1$ and $w_{u, 1}=w_{u, 2}=0$. Using these weights within Eqn. 19 to generate the standard deviations of $y_{t}$ and $u_{t}$, the optimization of Eqn. 26 was then carried out subject to the constraints on the operating point. The economic objective value achieved for this case was $J_{m v}=635$, whereas a value of $J_{o p t}=746$ was obtained by using the proposed procedure. This difference can be quantified by the measure $\gamma=\frac{J_{o p t}-J_{m v}}{J_{o p t}} \times 100 \approx 15 \%$ which shows about 15 percent lost economic potential when using the minimum variance controller compared to the optimal case.

Fig. 8 presents the operating region of each of the process variables for both the minimum variance and optimal control cases. It is noted that although $y_{1}$ and $y_{2}$ have reduced variation in the minimum variance case, they are much further from the more profitable region that is located near the upper bounds of these variables. This suboptimal behavior can be explained as follows: when decreasing $\sigma_{y, 1}$ and $\sigma_{y, 2}$ relative to their values in the optimized case, the resulting trade-off is that the values of $\sigma_{u, 1}$ and $\sigma_{u, 2}$ are inflated, pushing the whole operating point far from the optimal region. It is noted that in Fig. 8, both $u_{1}$ and $u_{2}$ are against their upper bounds for the minimum variance case.

Fig. 9 presents the closed-loop disturbance impulse response of each of the process variables in the case of minimum variance control. Comparing against the responses in the optimal case (Fig. 7), it is noted that although deadbeat control is achieved, the variation has indeed been transferred from the process variables, $y_{1}$ and $y_{2}$, to the controller outputs, $u_{1}$ and $u_{2}$. These observations are consistent with those in earlier works, which concluded that minimum variance control is often undesirable because of a variety of ill effects caused to transferring variability to the controller outputs [8].

\subsection{3-input 3-output Control Problem}

The second example that is now considered is a modification of the version of the Shell control problem examined by Maciejowski[22]. The continuous-time plant and disturbance transfer functions, $H(s)$ and $N(s)$, for this system are given by

$$
H(s)=\left[\begin{array}{lll}
\frac{4.05 e^{-27 s}}{50 s+1} & \frac{1.77 e^{-28 s}}{60 s+1} & \frac{5.88 e^{-27 s}}{50 s+1} \\
\frac{5.39 e^{-18 s}}{50 s+1} & \frac{5.72 e^{-14 s}}{60 s+1} & \frac{6.9 e^{-15 s}}{40 s+1} \\
\frac{4.38 e^{-20 s}}{33 s+1} & \frac{4.42 e^{-22 s}}{44 s+1} & \frac{7.2 e^{-s}}{19 s+1}
\end{array}\right]
$$

and

$$
N(s)=\left[\begin{array}{ll}
\frac{1.2}{45 s+1} & \frac{1.44}{40 s+1} \\
\frac{1.52}{25 s+1} & \frac{1.83}{20 s+1} \\
\frac{1.14}{27 s+1} & \frac{1.26}{32 s+1}
\end{array}\right]
$$

Two measured disturbances were considered: the first, $d_{1}$ is a step-type disturbance with a mean time between value changes of 100 minutes, while the second, $d_{2}$ was a load disturbance whose value changed only every several days and furthermore only took on values from the set $\{-0.25,0,0.25\}$. Disturbance 1 is treated as a stochastic disturbance in the framework of Eqn. 2 and so feedforward control is implemented to reject its effects on the outputs. 
Changes in the second disturbance are both large in magnitude and infrequent, so it is proposed to deal with this disturbance by changing the overall system operating point in an optimal way, whenever the disturbance level changes.

Several operational restrictions for this system are now described. The first two outputs are required to keep a mean value equal to their set-points $y_{1}^{*}=y_{2}^{*}=0$, while the third output is free to vary within its bounds. All three system inputs are free to vary within their bounds. The inputs and outputs are bounded as follows:

$$
\begin{aligned}
& -0.5 \leq u_{t, i} \leq 0.5, i=1 \ldots 3 \\
& -0.5 \leq y_{t, i} \leq 0.5, i=1 \ldots 3
\end{aligned}
$$

However, the constraints on $u_{t}$ correspond to limits of valve movement and are treated as hard constraints, whereas the bounds on each output $y_{t, i}$ can be violated up to $5 \%$ of the time.

Other than these specifications, no variable within this system has been assigned a cost or revenue, except for $u_{3}$ which is directly related to the amount of steam that is used in the reboiler. Since this is the only variable with economic value, the relative cost of $\bar{u}_{3}$ can be assigned to be 1 , and the objective function for this system can be written

$$
\max J=-\bar{u}_{3} .
$$

As indicated, the optimization of Eqn. 11 was carried out three times, once for each potential value of $d_{2}$. To handle the different magnitudes of $d_{2}$, the last constraint in Eqn. 11 was modified to become $\left(\bar{y}-y_{o}\right)=K\left(\bar{u}-u_{o}\right)+K_{d} d_{2}$, where steady state gain $K_{d}$ was determined according to $N\left(z^{-1}\right)$. Two cases were considered: Case 1 used the tuning determined by the algorithm in Fig. 5, while in Case 2, the controller was heuristically tuned, using the weights $w_{y, 1}=w_{y, 2}=w_{y, 3}=w_{u, 1}=w_{u, 2}=1$, and $w_{u, 3}=10$, where a large weight was associated with input $u_{3}$ because of this variable's relation to the economic objective.

To proceed with the procedure of Sec. 4.1, the transfer functions of Eqns. $27 \& 28$ were first discretized using an impulse-response invariant approximation with controller time step $T_{c}=1 \mathrm{~min}$, then further transformed to finite impulse response form. The plant impulse response model contained 200 matrix coefficients, while the control horizon was selected as 150 time steps. This meant that the closed-loop disturbance impulse response was computed for 350 time steps.

The weights in Eqn. 16 were varied in the range $10^{-2}$ to $10^{2}$ to create a data set of approximately 16,000 points in $\sigma$ space. Using the gpca algorithm, it was determined that the use of 9 linear subspaces provided a good approximation of the original data. Each subspace obtained via gpca was an unbounded hyperplane in 5 dimensions, and so the kDOPs method was used to bound each hyperplane in 242 directions. Then, the optimization problem of Eqn. 11 with objective function Eqn. 31 was solved on each of the bounded subspaces, the solutions for each subspace compared, and the overall optimal operating point and process variable standard deviations selected.

Because of the high dimensionality and sparse distribution of sample points in $\sigma$ space, to benchmark if the controller weights identified using the piece-wise linear approximation of the $\sigma$ surface was adequate, it was decided to use a neural network to map the space of $\sigma$ to the space of controller weights. A single hidden layer network with 20 nodes was trained on the sampled $\sigma$ data and was used to find the weights $\left(w_{y}, w_{u}\right)$ based on the optimal $\left(\sigma_{y}, \sigma_{u}\right)$. With the weights determined in this manner, an objective function within $1 \%$ of the original optimum was achieved, giving evidence that the piecewise linearization procedure of Fig. 5 provides an acceptable approximation to the $\sigma$-space.

Fig. 10 presents the controller weights used in each the optimal and heuristic tuning cases, as well as the resulting standard deviations of the process variables. While in the heuristic tuning case, low variation is achieved for variable $u_{3}$, it is seen from the optimal tuning that the most important variables (in terms of reducing their variation) are $y_{3}$ and $u_{1}$. In fact, for these variables the heuristically tuned controller does relatively poorly.

Next a simulation of the system described by Eqns. 27 \& 28 was carried out in Matlab Simulink. The simulation was run for $10^{4} \mathrm{~min}$ for both the optimally tuned and heuristically tuned cases; in each case, the operating points determined during the optimization were implemented according to the level of $d_{2}$, where this disturbance changed level approximately once every 2.2 days. Fig. 11 shows the results of the simulation trials. It can be seen that disturbance $d_{1}$ has relatively frequent fluctuation compared to $d_{2}$, supporting the decision to treat this variable within the stochastic framework.

The motivation for the relatively high weights given to $w_{y, 3}$ and $w_{u, 1}$ in the case of the optimal tuning can be observed from Fig. 11. In both the optimally and heuristically tuned cases, it appears that the constraints on these 
variables are preventing further decrease in the level of $u_{3}$. In Case 1, the optimal tuning assigns low variance to $u_{1}$ allowing it to be closer to the constraint boundary, which permits $u_{3}$ to take on relatively low values. However, in the case of heuristic tuning, variable $u_{1}$ is making frequent large magnitude and sudden changes that require this variable's mean value to be held far away from its constraint boundary. This in turn limits the flexibility provided to variable $u_{3}$, thus reducing the achievable performance in this heuristically tuned case. The mean value of the objective in Eqn. 31 achieved for the case of optimized tuning was $J_{o p t}=0.29$, while for the heuristically tuned case, it was only $J_{m v}=0.19$. Computing the lost economic potential as defined in the previous example, it is seen that the heuristic tuning case performs $\gamma=\frac{J_{o p t}-J_{m v}}{J_{o p t}} \times 100 \approx 34 \%$ worse than the case where the optimal trade-offs are made.

In some cases, the amount of variability exhibited by the outputs of the optimal case may not be acceptable for a certain variable. Whereas in traditional controller design, this would have to be handled via trial and error adjustment of weights within the controller's objective function, in contrast, the optimization formulation of this work allows that the standard deviation of any process variable can be hard-capped simply by the addition of a constraint to the optimization problem in Eqn. 11. The ability to more directly state process requirements and economic objectives within the framework proposed in this paper (and similar methods) is an advantage compared to traditional hierarchial operating point selection and controller tuning methods.

Remark 5.1. Though the potential of the proposed approach is demonstrated on square systems, it is a generalized method applicable to non-square systems as well. If the multivariate finite response models of the process and disturbance behaviour are available, then an optimization problem described in equation 11 could be formulated and solved using the proposed approach for multivariate systems under additive disturbances.

\section{Conclusion}

A novel approach for economical optimization of constrained multivariate control systems has been introduced. The proposed method requires plant, disturbance, and constraint information, but returns an estimate of both the optimal achievable economic performance, as well as the minimum backed-off operating point and controller weights that can provide this performance level. The two case studies presented in this work showed that the new method can be used to derive higher economic benefit compared to other methods for controller design.

Currently, the proposed approach assumes the existence of a multivariate predictive controller and obtains the controller weights by solving the minimum backed-off operating point selection problem. This method could be extended for identification of a MIMO controller wherein the controller structure and parameters are functions of the decision variables in the optimization problem solved in this work. Though this could leading to a complex problem compared to the one considered, with a proper framework, synthesis of MIMO controller could be achieved. Another important requirment/assumption of the proposed method is the availability of finite impulse response plant and disturbance model. Future work is directed towards development of algorithms to obtain a reasonable model of the plant and disturbances from routine operating data with minimal excitations that could be used in obtaining the FIR model of the process.

\section{References}

[1] M. Forbes, M. Guay, J. Forbes, Control design for first-order processes: shaping the probability density of the process state, Journal of process control 14 (4) (2004) 399-410.

[2] C. Pryor, Autocovariance and power spectrum analysis derive new information from process data, Control Engineering 29 (11) (1982) 103106.

[3] T. J. Harris, Assessment of control loop performance, The Canadian Journal of Chemical Engineering 67 (5) (1989) 856-861.

[4] M. Jelali, An overview of control performance assessment technology and industrial applications, Control Engineering Practice 14 (5) (2006) 441-466.

[5] B. Huang, S. Shah, H. Fujii, The unitary interactor matrix and its estimation using closed-loop data, Journal of Process Control 7 (3) (1997) 195-207.

[6] T. Harris, F. Boudreau, J. MacGregor, Performance assessment of multivariable feedback controllers, Automatica 32 (11) (1996) $1505-1518$.

[7] B.-S. Ko, T. F. Edgar, Performance assessment of multivariable feedback control systems, Automatica 37 (6) (2001) $899-905$.

[8] B. Huang, S. L. Shah, Performance assessment of control loops: theory and applications, Springer, Berlin, 1999.

[9] L. T. Narraway, J. D. Perkins, G. Barton, Interaction between process design and process control: economic analysis of process dynamics, Journal of Process Control 1 (5) (1991) 243-250. 
[10] L. T. Narraway, J. D. Perkins, Selection of process control structure based on linear dynamic economics, Industrial \& Engineering Chemistry Research 32 (11) (1993) 2681-2692.

[11] D. J. Chmielewski, A. M. Manthanwar, On the tuning of predictive controllers: Inverse optimality and the minimum variance covariance constrained control problem, Industrial \& Engineering Chemistry Research 43 (24) (2004) 7807-7814.

[12] J.-K. Peng, A. M. Manthanwar, D. J. Chmielewski, On the tuning of predictive controllers: The minimum back-off operating point selection problem, Industrial \& Engineering Chemistry Research 44 (20) (2005) 7814-7822.

[13] M. Nabil, S. Narasimhan, S. Skogestad, Economic back-off selection based on optimal multivariable controller, in: Proceedings of the 8th IFAC International Symposium on Advanced Control of Chemical Processes, 2012, pp. 792-797.

[14] C. Zhao, Y. Zhao, H. Su, B. Huang, Economic performance assessment of advanced process control with lqg benchmarking, Journal of process control 19 (4) (2009) 557-569.

[15] R. Vidal, Y. Ma, S. Sastry, Generalized principal component analysis (gpca), Pattern Analysis and Machine Intelligence, IEEE Transactions on 27 (12) (2005) 1945-1959.

[16] R. Vidal, Generalized principal component analysis (gpca): an algebraic geometric approach to subspace clustering and motion segmentation, Ph.D. thesis, University of California Berkeley (2003).

[17] J. T. Klosowski, M. Held, J. S. Mitchell, H. Sowizral, K. Zikan, Efficient collision detection using bounding volume hierarchies of k-dops, Visualization and Computer Graphics, IEEE Transactions on 4 (1) (1998) 21-36.

[18] J. F. MacGregor, T. J. Harris, J. Wright, Duality between the control of processes subject to randomly occurring deterministic disturbances and arima stochastic disturbances, Technometrics 26 (4) (1984) 389-397.

[19] L. Ljung, T. Glad, Modeling of dynamic systems.

[20] B.-S. Ko, T. Edgar, Performance assessment of cascade control loops, AIChE Journal 46 (2) (2000) $281-291$.

[21] R. Wood, M. Berry, Terminal composition control of a binary distillation column, Chemical Engineering Science 28 (9) (1973) $1707-1717$.

[22] J. M. Maciejowski, Predictive control with constraints, Prentice Hall, 2002. 
(a)

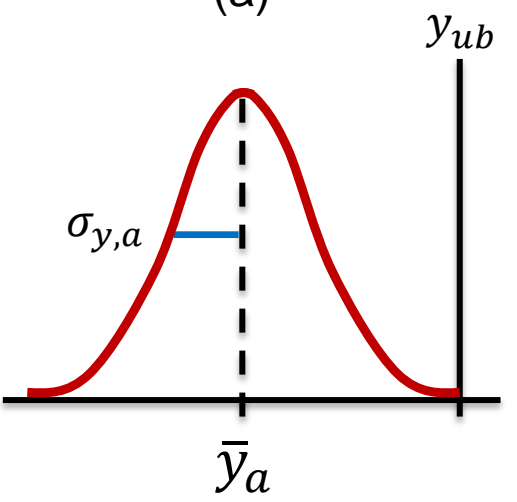

(b)

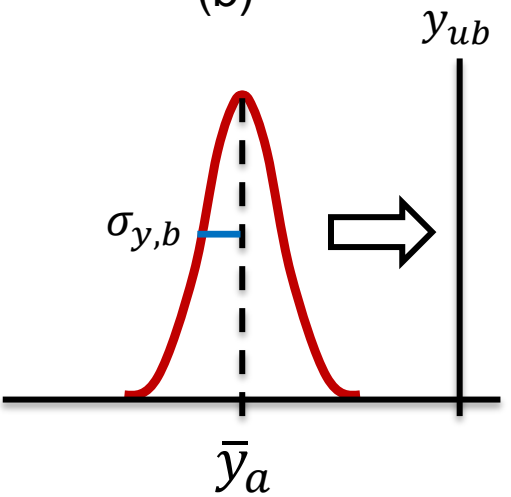

(c)

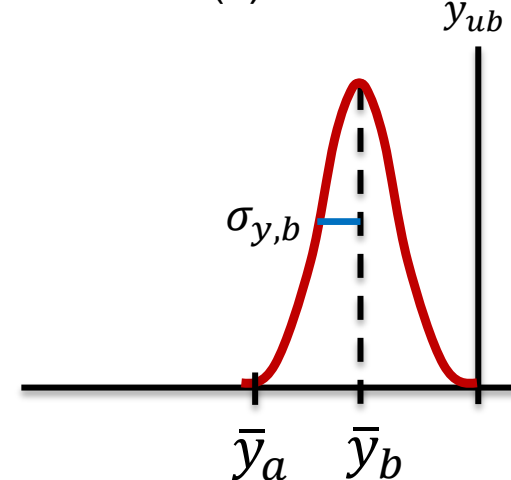

Figure 1: Variance and operating point interaction in SISO systems

(a)

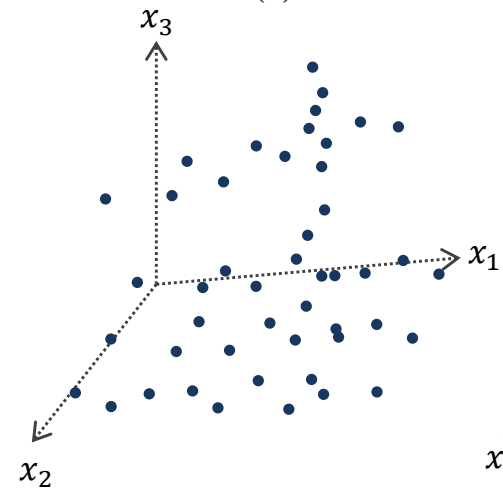

(c)
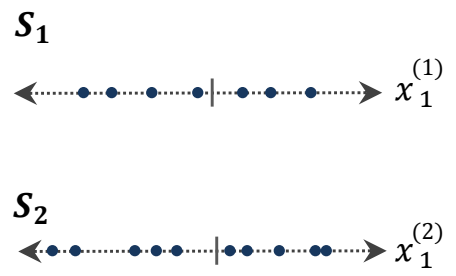

(b)
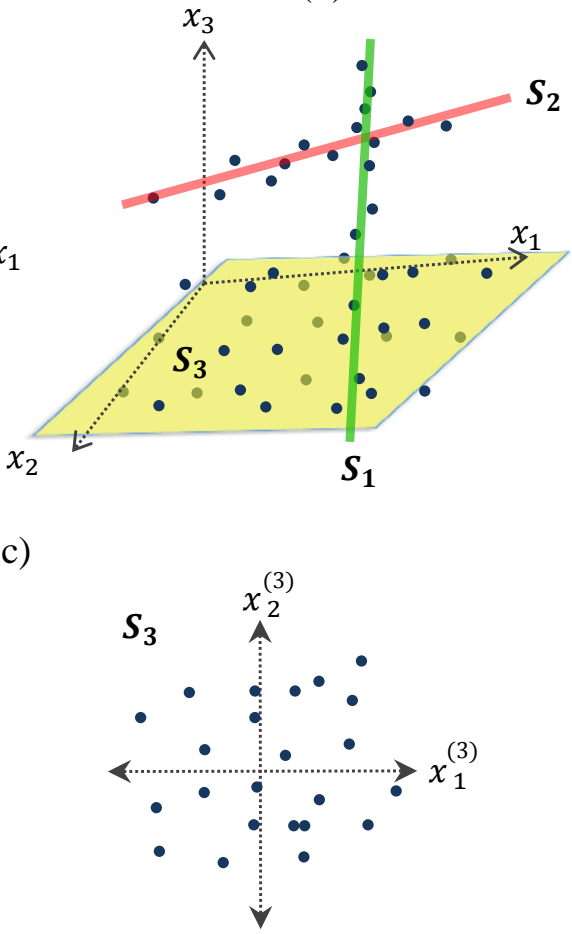

Figure 2: Illustration of gpca: (a) original data set; (b) gpca-determined subspaces; (c)projection of points onto low-dimensional subspaces 


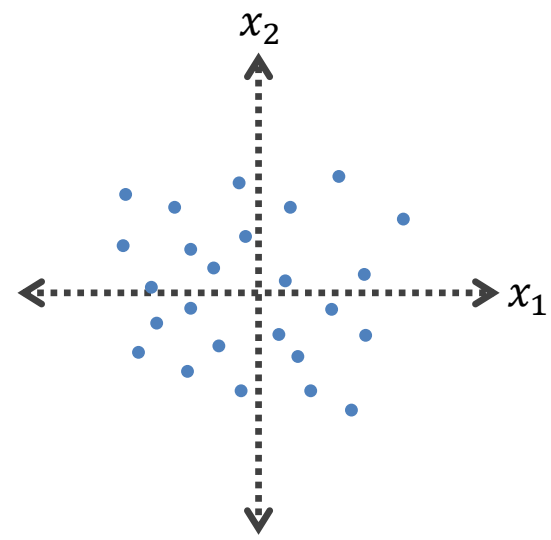

(a) Position axes on data mean

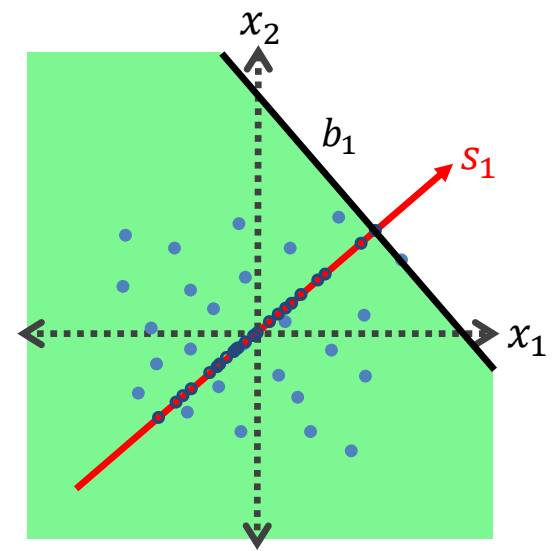

(c) Bound data by hyperplane $b_{1}$ which is normal to $S_{1}$

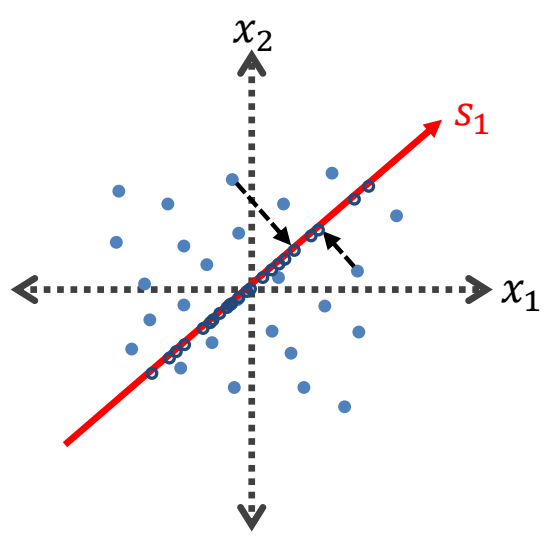

(b) Project data onto direction $s_{1}$

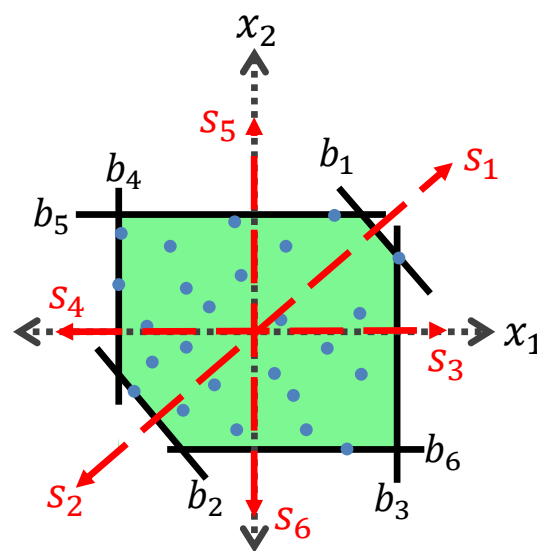

(d) Create pairs $\left(s_{i}, b_{i}\right), i=2 \ldots k$ to complete bounding box

Figure 3: Illustration of k-DOP construction 


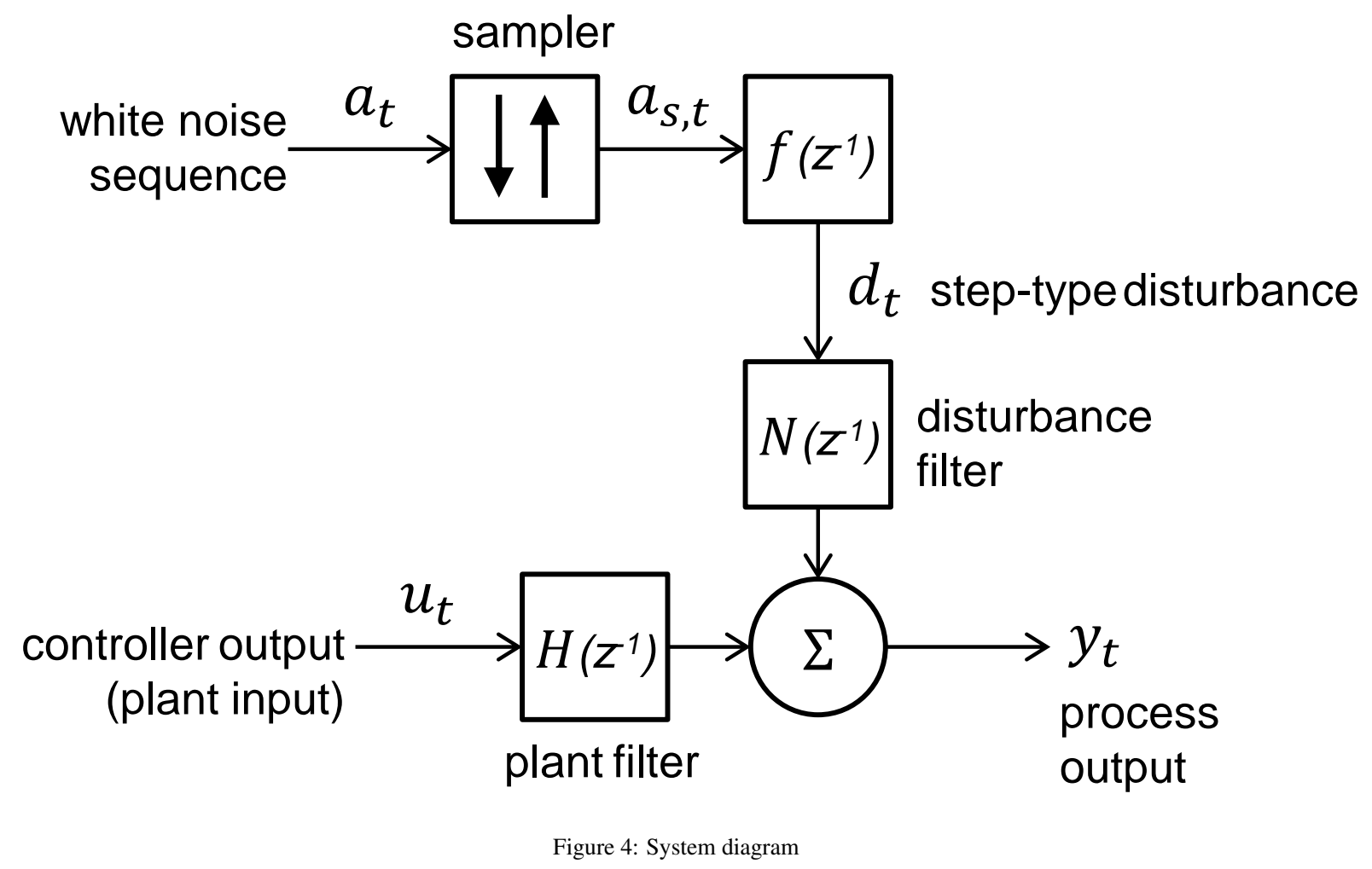




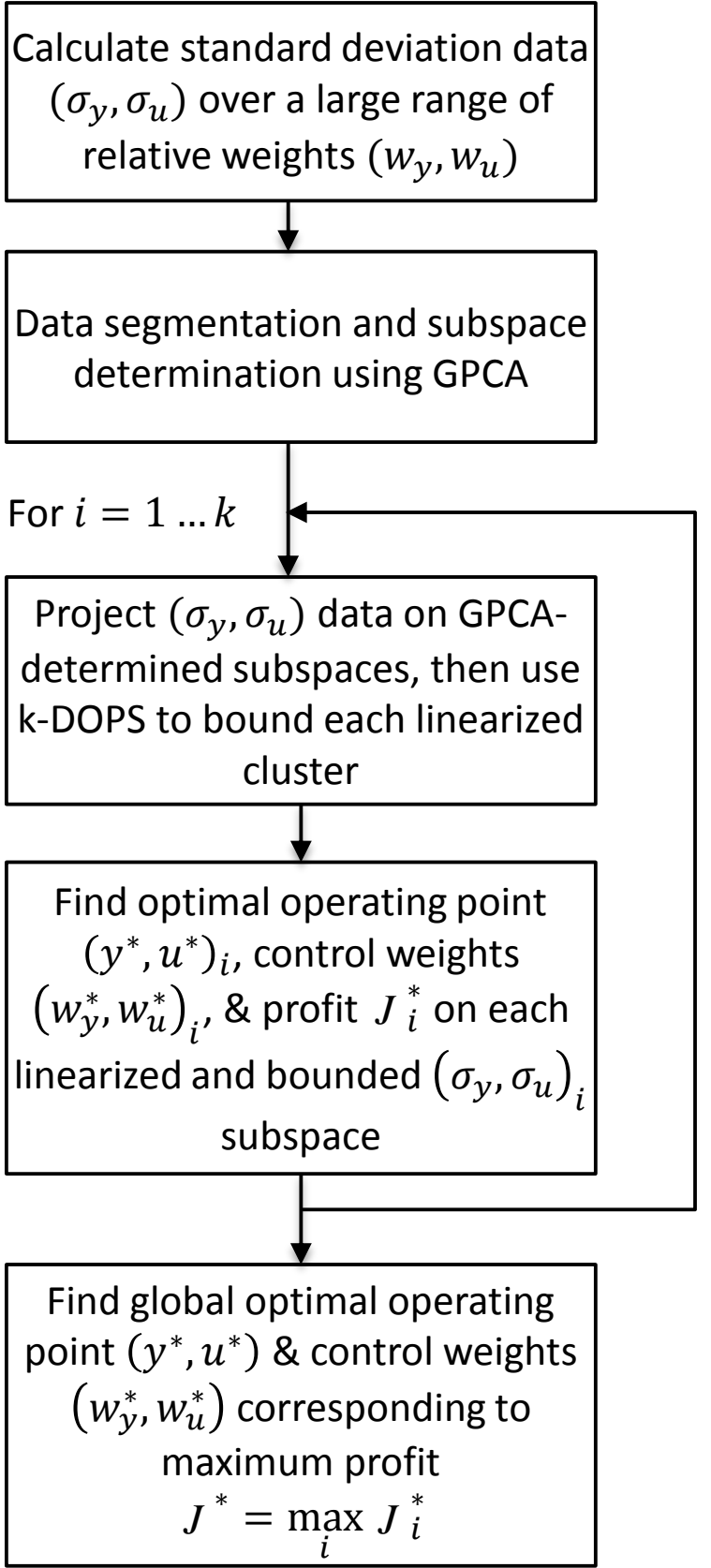

Figure 5: Operating point and controller weights selection algorithm 
(a)

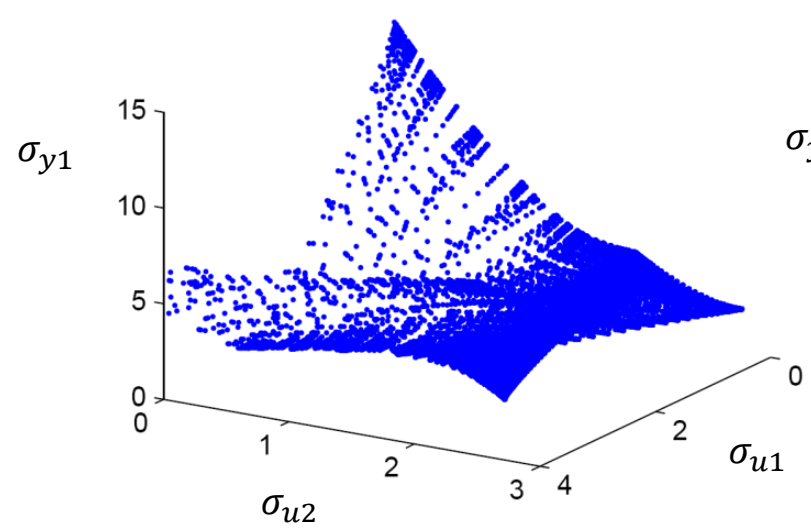

(c)

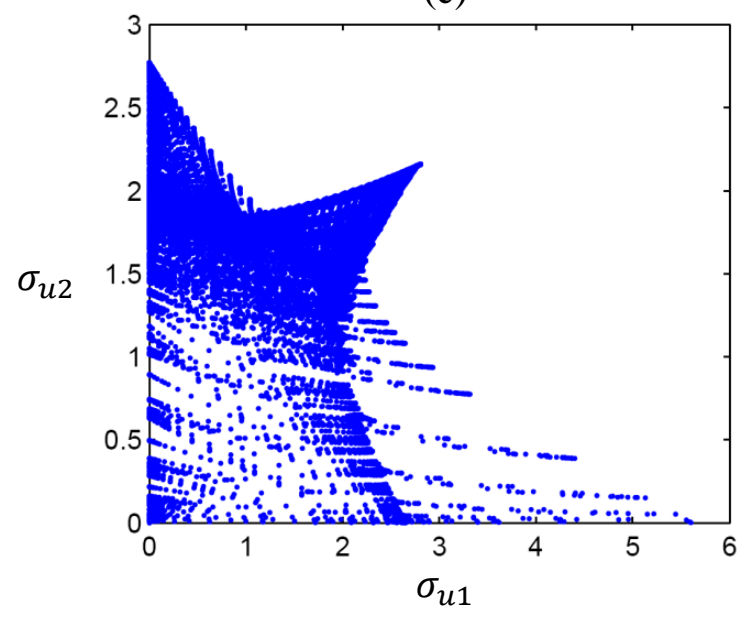

(b)

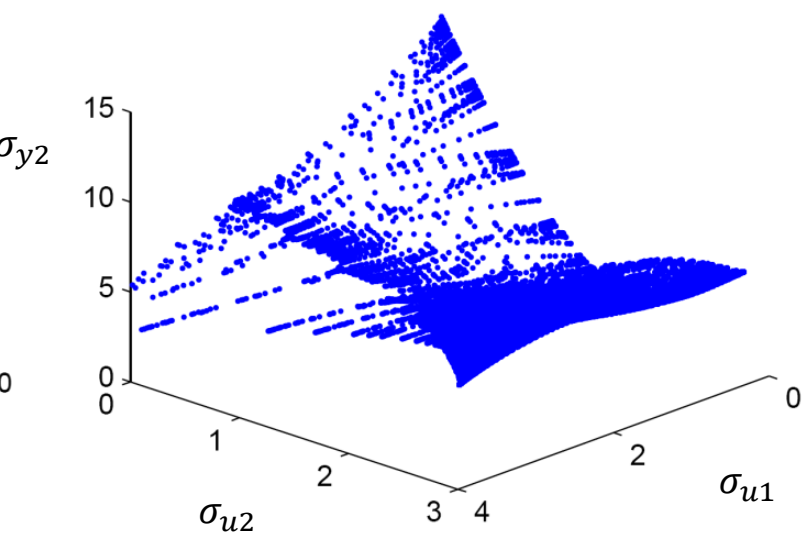

(d)

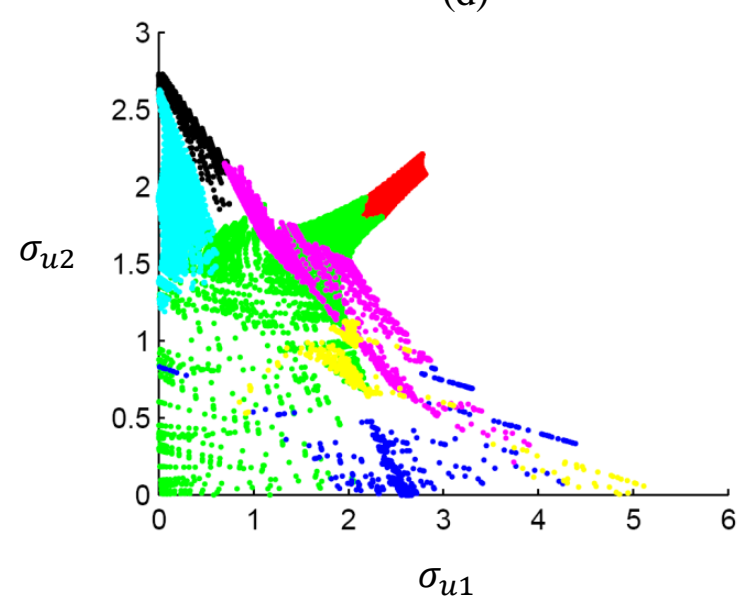

Figure 6: Wood \& Berry column: Subspaces of the $\left(\sigma_{u, 1}, \sigma_{u, 2}, \sigma_{y, 1}, \sigma_{y, 2}\right)$ space 

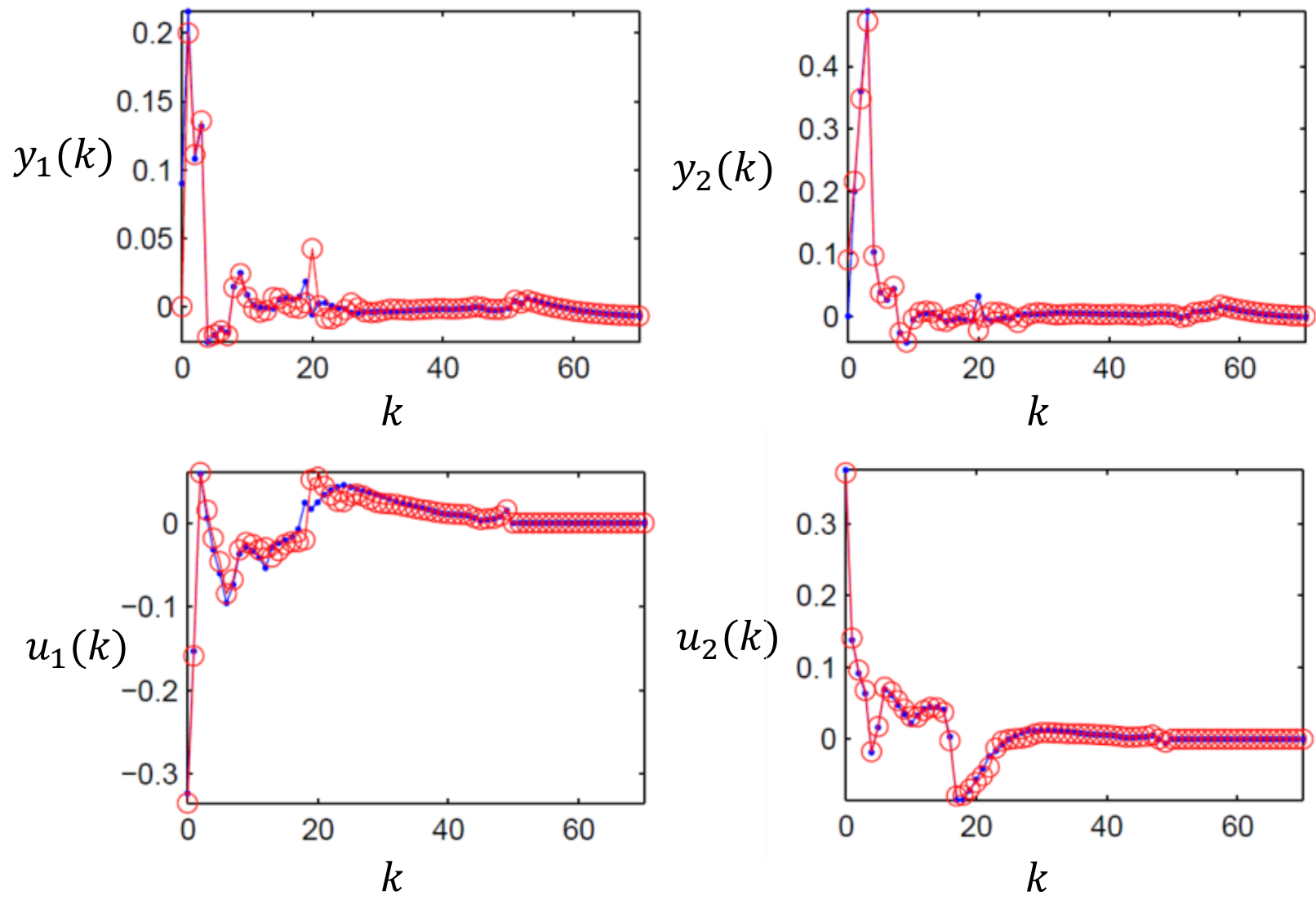

Figure 7: Wood \& Berry column: Disturbance impulse responses under the optimized controller

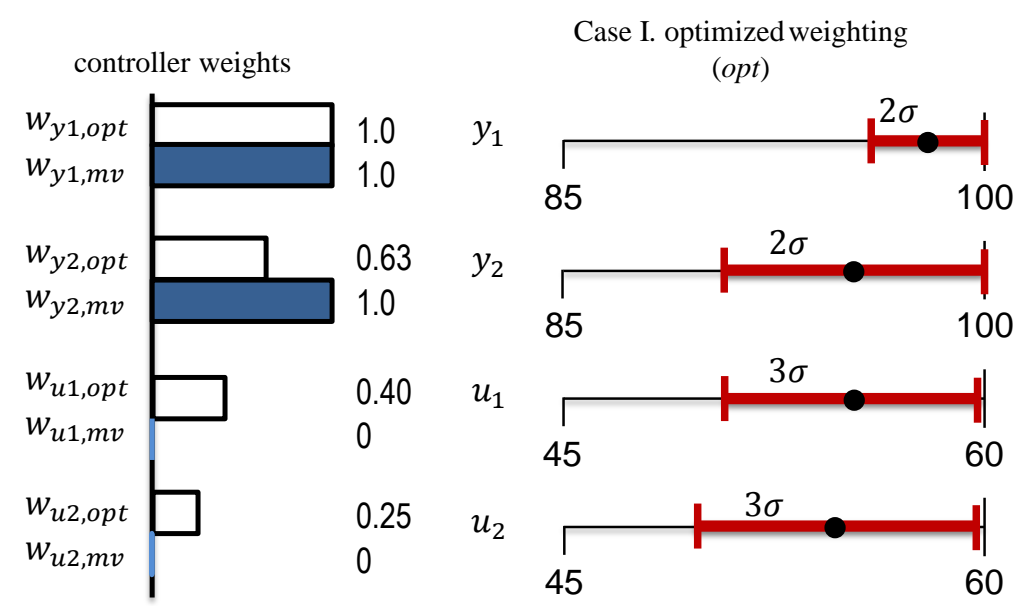

Case II. weighting for minimum output variance

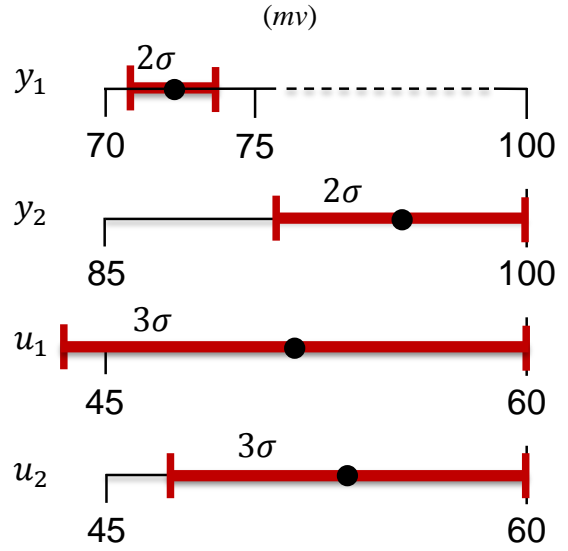

Figure 8: Wood \& Berry column: controller weights and resulting dynamic operating regions 

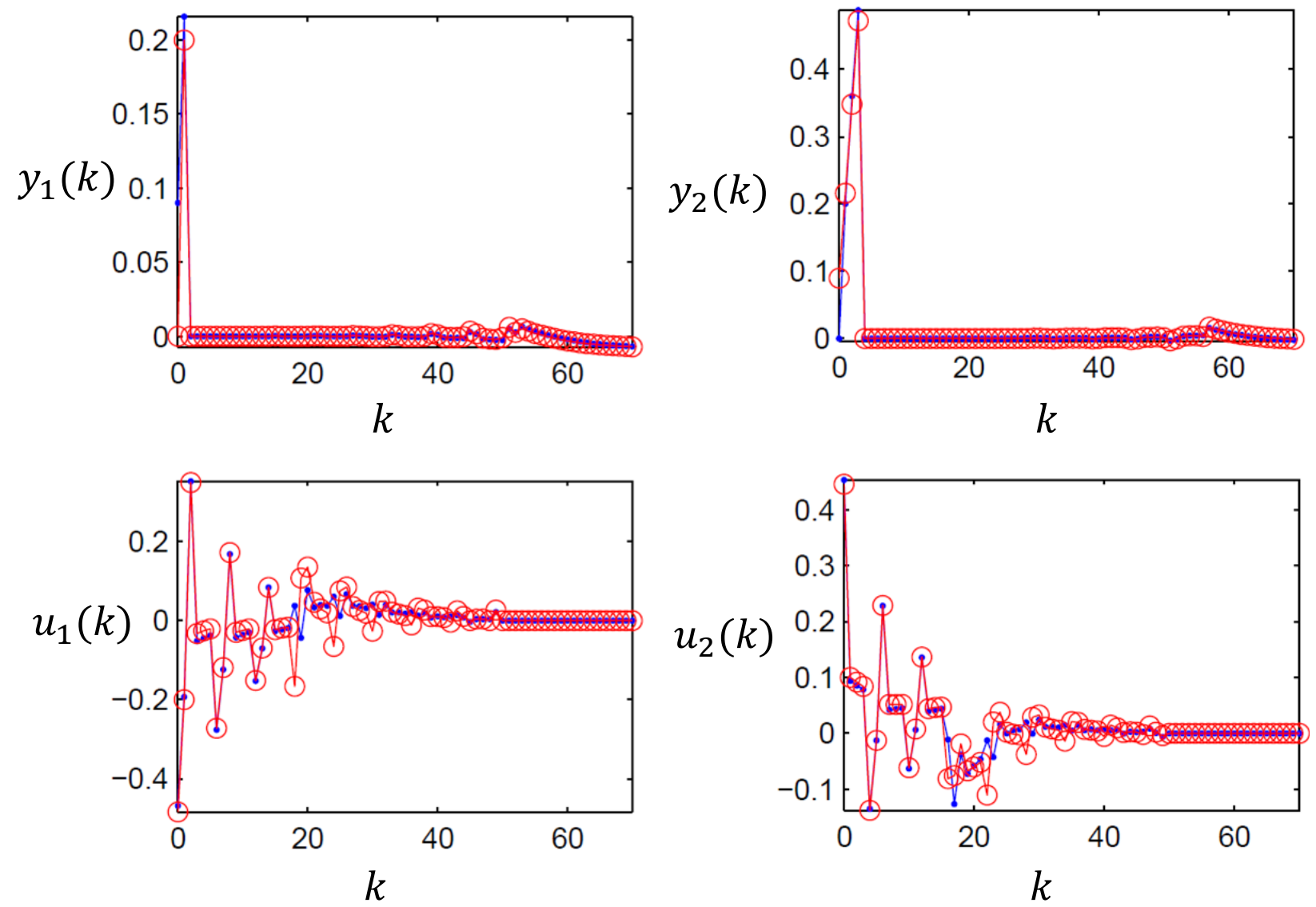

Figure 9: Wood \& Berry column: Disturbance impulse responses under minimum variance control 
(a)

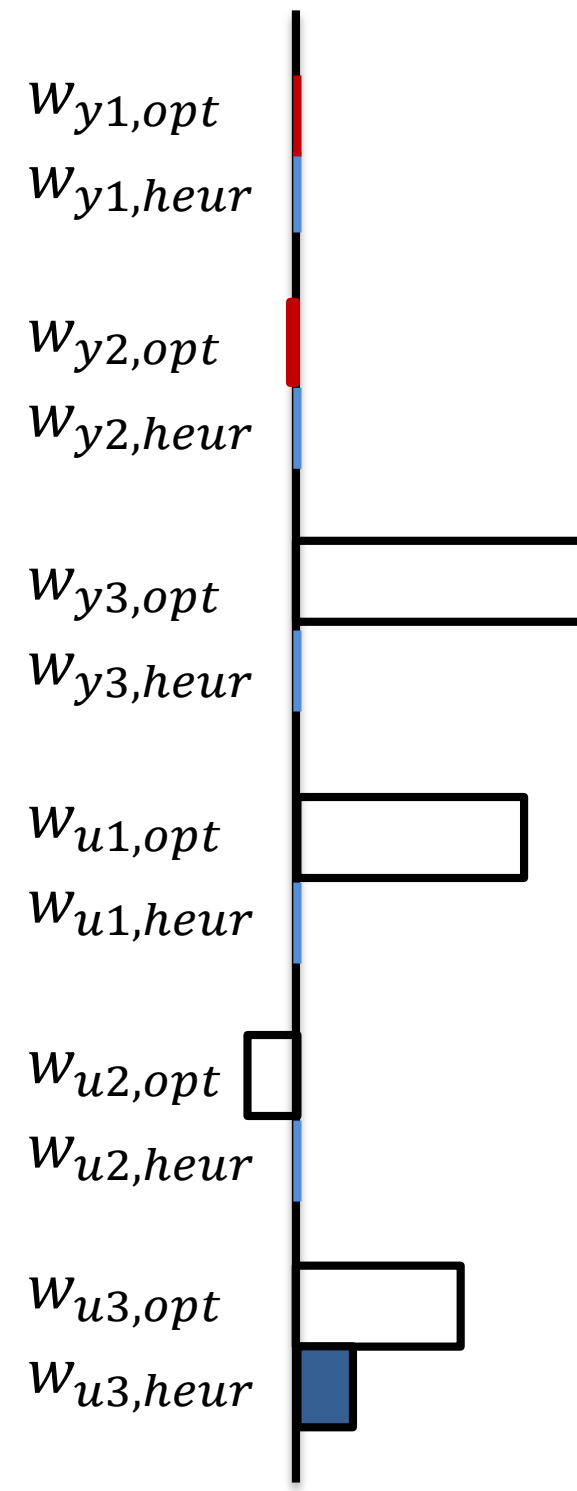

(b)

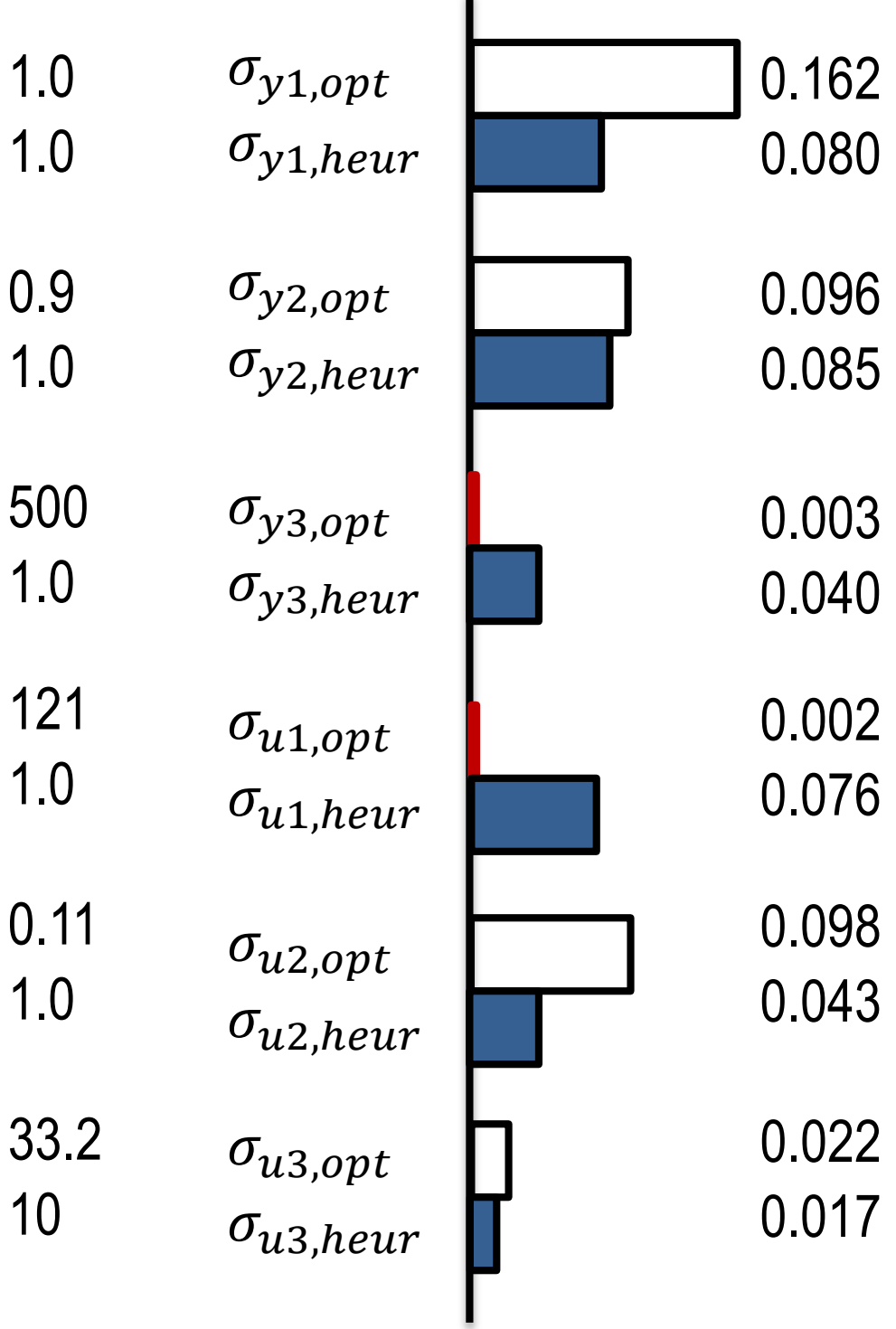

Figure 10: Shell control problem: (a) controller weights (logarithmically scaled bars) and (b) resulting process variable standard deviations (linear scaled bars) 


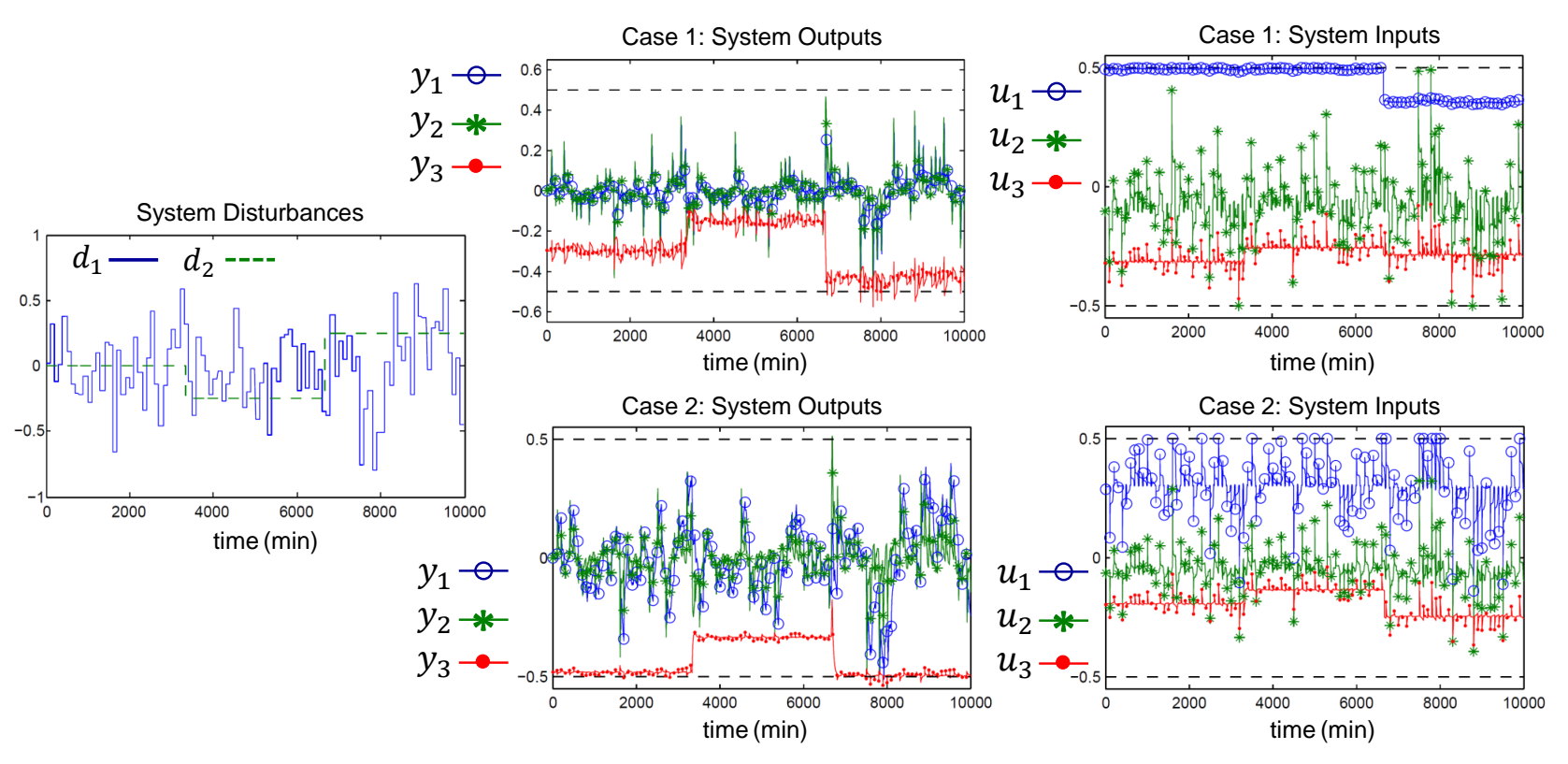

Figure 11: Shell control problem: Case 1 - optimal operating point and controller tuning; Case 2 - heuristical controller tuning 